\title{
Application of Fascia of Temporal Muscle and Cartilage of Auricular Tragus in Myringoplasty in Children
}

\section{Ivan Baljosevic $^{1 *}$, Slavisa Antic ${ }^{2}$ and Stefan Popovic ${ }^{1}$}

${ }^{1}$ ORL Department, Mother and Child Health Institute, Belgrade

${ }^{2}$ Community Health Centre "Dr Simo Milosevic" Čukarica, Belgrade

*Corresponding Author: Ivan Baljosevic, ORL Department, Mother and Child

Health Institute, Belgrade.
Received: August 19, 2021

Published: September 06, 2021

(C) All rights are reserved by Ivan Baljosevic., et al.

\section{Abstract}

Introduction: Perforation of tympanic membrane in children may be cause of recurrent midlle ear infection and loss of hearing. Objective: The aim of this study is to analyze application of different reconstructive materials in surgical technique-miringoplasty. Methods: We performed 88 myringoplasty in 76 children during the period of July 2010. and July 2018. Age, gender, size and site of perforation, status of the contra lateral ear, underlying cause of the perforations, surgical technique, preoperative and postoperative hearing levels and postoperative complications were recorded. In performing myringoplasty we used fascia of temporal muscle and cartilage of auricular tragus.

Results: In 43 (48\%) patients we used fascia of temporal muscle and in 45 (52\%) cartilage of auricular tragus. Graft success was defined as an intact eardrum at 12 months postoperatively and an improvement in perception of air-bone gap of $10 \mathrm{~dB}$, which we recorded in 73 (83\%) cases. Reperforations were noted in $12(13 \%)$ patients, in 8/43 (18,6\%) cases where we used temporal fascia and in $4 / 45(9,3 \%)$ cases with tragal cartilage. Retraction of tympanic membrane was recorded in $3 / 43$ (4\%) cases with temporal fascia.

Conclusion: Myringoplasty is a reasonably successful method with good functional results for the pediatric patients. Risk factors for surgical success were size of perforation and pathological conditions of contra lateral ear. Tragal cartilages give better results in cases with bilateral perforations because possibility of retractions and reperforations is smaller.

Keywords: Myringoplasty; Children; Surgical Technique

\section{Introduction}

Perforations of the eardrum, caused by pathological processes, can as well cause significant discomfort on children, such as: frequent and repeated infections of the middle ear, consequent hearing loss, followed by all these problems, very possible difficult speech development and slow intellectual development. These children must avoid swimming, and on the other hand, they often turn to a doctor for treatment. Therefore, it is necessary to close the perforation on the eardrum with surgical treatment and improve hearing. This improves the quality of life of children, their intellectual development, as well as speech development. In the literature, the success of tympanoplasty type I (myringoplasty) ranges between 56 and 94\% [1,2]. However, some authors do not recommend performing tympanoplasty on children [3]. These authors believe that the success of surgery on children is much weaker than on adults and link this weaker success to a number of factors that include frequent respiratory infections, relatively poor Eustachian 
tube function, weakened immunity, narrow external ear canal and technical difficulties, difficult postoperative monitoring and inability to cooperate with the patient.

\section{The Goal of the Work}

The aim of this paper is to point out the advantages and disadvantages of using different reconstructive materials in myringoplasty in children.

\section{Materials and Methods}

The research was performed as a controlled clinical study at the ORL department of the Mother and Child Health Institute of Serbia, in Belgrade, which is a tertiary clinical institution for the treatment of children. In the period from July 2010 to July 2018, 88 myringoplasties were performed in 76 children due to perforations on the eardrum. The children ranged in age from 6 to 16, with an average age of 11.9. The diagnosis was made on the basis of clinical symptoms of the disease, otoscopic and Oto microscopic examination. All children underwent tonal audiometry on the Inter-acoustics AD device. Using Oto-microscopy, perforation was classified as "small" when it was less than $25 \%$ of the eardrum, "medium" between 25 and $50 \%$ and "large" - more than $50 \%$ of the eardrum surface.

The perforation site is classified as anterior, posterior, and total perforation. Patients with middle ear cholesteatoma, as well as patients with auditory chain damage, were excluded from the study. Acute ear infection was considered a contraindication for surgery. Surgical treatment was performed under an operating microscope, followed by retro-auricular and endaural approach under general anesthesia, underlay technique. The fascia of the temporalis muscle and the cartilage of the tragus of the auricle were used in the reconstruction. Successful perforation closure was defined when there was an intact tympanic membrane one year after surgery. Hearing improvement success is defined as an improvement of 10 decibels or more at two selected frequencies compared to preoperative airborne sound conduction. Preoperative and postoperative critical (limit) values were measured at 500, 1000 and $2000 \mathrm{~Hz}$. The analysis of the significance of the difference, ie, the Chi (Hi) square test, was applied and the statistical significance between the two groups of patients was determined.

Results
Out of the 76 children, 42 (55\%) were male and 34 (45\%) were female. The duration of the disease was from 6 months to 5 years, average 2.3 years. The time of postoperative follow-up of patients was from 6 months to 2 years (average 12 months). 8 (10.5\%) children had bilateral perforation of the eardrum. Out of the $88 \mathrm{op}$ erated cases, $17(15 \%)$ were under 7 years of age at the time of surgery, 44 (50\%) were between 8 and 12 years old, and 31 (35\%) were between 12 and 16 years old.

Preoperative anterior perforation was found in 21 (24\%) cases, posterior in 17 (19\%), central in 29 (32\%) and total in 22 (25\%). Small perforation was found in 17 (19\%), medium was found in 34 (38\%) and large in 38 (43\%) children. All patients had dry ears at the time of surgery. 26 (29\%), 15 (17\%) after recurrent acute otitis and 47 (54\%) after chronic otitis had perforation after ventilation tube extraction (Table 1). Tonal audiometry preoperatively diagnosed mild conductive hearing damage to all children, with a difference between air and bone sound conductivity (cochlear reserve) of 10 to $20 \mathrm{~dB}$. In $35 \%$ of patients before performing tympanoplasty, adenoid vegetations were surgically removed due to other indications (secretory otitis, hypertrophy, recurrent adenoiditis). Myringoplasty surgery was performed and the defect on the eardrum was closed with reconstruction material. In 43 (48\%) children we used the fascia of the temporalis muscle, and in 45 (52\%) the cartilage taken from the tragus. In 73 (83\%) cases the eardrum was intact in the follow-up period, re-perforation was present in 12 (13\%) patients, and eardrum retraction in 3/43 (4\%) in whom fascia was used. $73(83 \%)$ patients had a cochlear reserve of less than 10 dB. Reperforation was present in 12 (13.6\%) cases, when reconstruction was performed with fascia in $8 / 43$ (18.6\%) and tragus cartilage in $4 / 45$ cases $(9.3 \%)$. These were children younger than 10 years in $6(50 \%)$ cases, without removed adenoids in $4(33 \%)$. These were large perforations of the eardrum in all $12(100 \%)$ and in cases when they had a perforation on the other ear in 6/8 (75\%). Reperforations after fascia reconstruction were larger than $5 \mathrm{~mm}$ in all cases and required reoperation. In the second act, we always used cartilage taken from the tragus. Reperforations after cartilage reconstruction were less than $3 \mathrm{~mm}$ in all cases, so we estimated that no new surgery should be performed. It was found that there was no statistically significant difference in the occurrence of reperforation, when fascia or tragus were used $(p=0.24$, Chi square $=1.34$ ). 


\begin{tabular}{|l|c|c|c|}
\hline Site of perforations & $\begin{array}{c}\text { Total } \\
\text { numbers }\end{array}$ & $\begin{array}{c}\text { Success after } \\
\text { operation }\end{array}$ & \% \\
\hline Anterior & 21 & 19 & 90 \\
\hline Posterior & 17 & 16 & 94 \\
\hline Central & 29 & 25 & 86 \\
\hline Total & 22 & 17 & 77 \\
\hline Size of Perforations & \multicolumn{3}{|l}{} \\
\hline Small & 17 & 17 & 100 \\
\hline Medium & 34 & 34 & 100 \\
\hline Large & 38 & 26 & 68 \\
\hline Cause of Perforations & \multicolumn{3}{|l}{} \\
\hline $\begin{array}{l}\text { After Extractions of Venti- } \\
\text { lation Tubes }\end{array}$ & 26 & 23 & 88 \\
\hline Recurrent Otitis & 15 & 11 & 73 \\
\hline Chronic Otitis & 47 & 40 & 85 \\
\hline
\end{tabular}

Table 1: Site, size and cause of perforations.

\section{Discussion}

Myringoplasty is an effective surgical procedure that can very successfully close the perforation on the eardrum. There are a number of different opinions about risk factors that may affect the success of type I tympanoplasty or myringoplasty on children. Many anatomical and functional factors can be mentioned as risk factors, such as the patient's age, size and location of perforation, condition of the other ear, graft material, surgical technique and preoperative hearing level $[3,4]$. It is recommended that tympanoplasty type I is not performed in children younger than 10 years, because the failure of the operation is greater in smaller children. Black [5] and MacDonald [6] report that success is much lower on children under 8 years of age. It is believed that the existing dysfunction of the Eustachian tube, repeated infections of the upper respiratory tract, irregular visits to check-ups and frequent reperforations of the eardrum are the dominant reasons for delaying the procedure until a certain age, which can vary between 10 and 14 years. Some authors believe that perforation of the eardrum is equivalent to a ventilation tube, and there is no need to hurry with closing [7].

On the other hand, Carr [8] and Caylan [1] believe that the patient's age has no effect on the success of myringoplasty. In our study, 6 (50\%) patients with reperforation were younger than 10 years, so it cannot be stated with certainty that the patient's age plays a dominant role in the success of the operation. Gianoli., et al.
[9] indicate that adenoidectomy performed before tympanoplasty reduces the risk of developing reperforation. In our results, 35\% of patients with reperforation had previously removed adenoid vegetations. This can be explained by the fact that children have frequent adenoid infections which, as a result, can be transmitted to the middle ear via the Eustachian tube and compromise the success of the operation. Certainly, the function of the Eustachian tube can be an important prognostic factor in the surgery of middle ear disease. No simple test can measure all aspects of Eustachian tube function. Some authors demonstrate that available tests have only limited prognostic values by showing that good Eustachian tube function can predict a good outcome, but that poor function does not necessarily predict a poor outcome $[10,11]$. It is very important to know the condition of the other, opposite ear, because if there is a deaf ear, existing perforation on that eardrum, otorrhea or weak auditory threshold as a bilateral pathology of the ear, then it indicates a serious dysfunction of the Eustachian tube.

Success has been found to be lower on children with bilateral perforations on the eardrum, retraction pocket, or secretory otitis $[1,4,12]$. And in our study, the results show that success is significantly better with patients that had healthy the other ear. In cases where they had a perforation on the other ear, reperforation occurred in 6/8 (75\%), which in turn can be associated with poorer eustachian tube function. Also, the size of the perforation on the eardrum can be a significant risk factor for the success of the operation. All cases, 12 of them (100\%), which we operated on again had perforations greater than $50 \%$ in the first act.

Different materials can be used as a material for the reconstruction of the tympanic membrane: carbon paper, adipose tissue of the auricle lobules, but the fascia of the temporalis muscle and the cartilage of the tragus are by far the most commonly used. Both the fascia of the temporalis muscle and the cartilage of the tragus belong to the easily accessible materials with low metabolic requirements and are suitable for closing the perforations on the tympanic membrane, regardless of the size of the perforation. Certainly, the fascia is a thinner and more elastic material and the results of hearing should be better than with the application of cartilage, which is thicker and more rigid, and it needs to be thinned to about $0.5 \mathrm{~mm}$ before it is placed behind the remains of the eardrum. As a harder material, cartilage is more difficult to perforate and provides prevention of retraction and possible consequent atelectasis. In our 
study, we registered 3 (4\%) retractions of the tympanic membrane in cases where the fascia was used as a reconstruction material. We installed a ventilation tube for these patients and the condition improved. Retraction, atelectasis and secretory otitis occur in 2 to $5 \%$ of operated patients in whom myringoplasty was performed with the fascia of the temporalis muscle $[7,13,14]$. This postoperative complication was not registered in patients in whom tragus cartilage was used.

Of the total number of cases in which we registered reperforations - 12, in 8 the fascia was used in the first operation, and in 4 cartilage. All newly formed perforations in children in whom fascia was used were larger than $5 \mathrm{~mm}$ and required reoperation. In the second act, we always used the cartilage of the tragus for reconstruction. In cases where cartilage perforation occurred, the size of the perforation itself was about $3 \mathrm{~mm}$, and did not require resurgical treatment. This can be explained by the fact that the fascia is a thinner, softer material that easily gives way during later infections of the middle ear. If we take the closure of the perforation as a measure of success, the percentage of success taken from the works of other authors is between 75 and 92\% [7] and in our study it was $83 \%$.

After performing myringoplasty, in a large number of cases, hearing improves. Various authors [15] most often describe: postoperative cochlear reserve from 0 to $10 \mathrm{~dB}$ in $49 \%$, from 0 to 20 $\mathrm{dB}$ in $87 \%$, from 0 to $30 \mathrm{~dB}$ in $96 \%$. In our study, all patients had a cochlear reserve of less than $20 \mathrm{~dB}, 73$ (83\%) less than $10 \mathrm{~dB}$.

\section{Conclusion}

Myringoplasty or tympanoplasty type I is a technique that can be successfully and safely performed on children with good functional results. Risk factors included for the success of surgical treatment are: the size of the perforation of the eardrum, as well as pathological changes in the other ear. Surgical treatment should be performed in the area of dry ear. Cartilage should be used to close bilateral perforations due to the lower degree of reperforation and retraction. Patients should be monitored for two years after surgery.

\section{Bibliography}

1. Caylan R., et al. "Myringoplasty in children: factors influencing surgical outcome". Otolaryngology-Head and Neck Surgery 118 (1998): 709-713.

2. Eisenbeis JF and Herrmann BW. "Areolar connective tissue grafts in pediatric tympanoplasty: a pilot study". American Journal of Otolaryngology 25 (2004): 79-83.
3. Lancaster JL., et al. "Pediatric tympanoplasty". The Journal of Laryngology and Otology 113 (1999): 628-632.

4. Collins WO., et al. "Pediatric tympanoplasty: effect of contralateral ear status on outcomes". Archives of Otolaryngology-Head and Neck Surgery 129 (2003): 646-651.

5. Black JH., et al. "An analysis of the results of myringoplasty in children". International Journal of Pediatric Otorhinolaryngology 31 (1995): 95-100.

6. MacDonald RR., et al. "Fasciaform myringoplasty in children". Archives of Otolaryngology--Head and Neck Surgery 120 (1994): 138-143.

7. Umapathy N and Dekker P. "Myryngoplasty: Is it worth performing in children?" Archives of Otolaryngology--Head and Neck Surgery 129 (2003): 1053-1055.

8. Carr MM., et al. "Success rates in paediatric tympanoplasty". Journal of Otolaryngology 30 (2001): 199-202.

9. Gianoli G., et al. "Pediatric tympanoplasty: The roleof adenoidectomy". Otolaryngology-Head and Neck Surgery 113 (1995): 380-386.

10. Tos M., et al. "Results of tympanoplasty in children after 15 to 27 years". Annals of Otology, Rhinology and Laryngology: SAGE Journals 109 (2000): 17-23.

11. Uyar Y., et al. "Tympanoplasty in pediatric patients". International Journal of Pediatric Otorhinolaryngology 70 (2006): 1805-1809.

12. Denoyelle F., et al. "Myringoplasty in children: predictive factors of outcome". Laryngoscope 109 (1999): 47-51.

13. Chandresekhar SS., et al. "Pediatric tympanoplasty: a 10-year experience". Archives of Otolaryngology--Head and Neck Surgery 121 (1995): 873-878.

14. Gaslin M., et al. "Pediatric cartilage interleave tympanoplasty". Otolaryngology-Head and Neck Surgery 134.2 (2007): 284288.

15. Vrabec J., et al. "Meta-analysis of pediatric tympanoplasty". Archives of Otolaryngology--Head and Neck Surgery 125 (1999): 530-534.

Volume 4 Issue 10 October 2021

(C) All rights are reserved by Ivan Baljosevic., et al. 